Provided for non-commercial research and education use. Not for reproduction, distribution or commercial use.

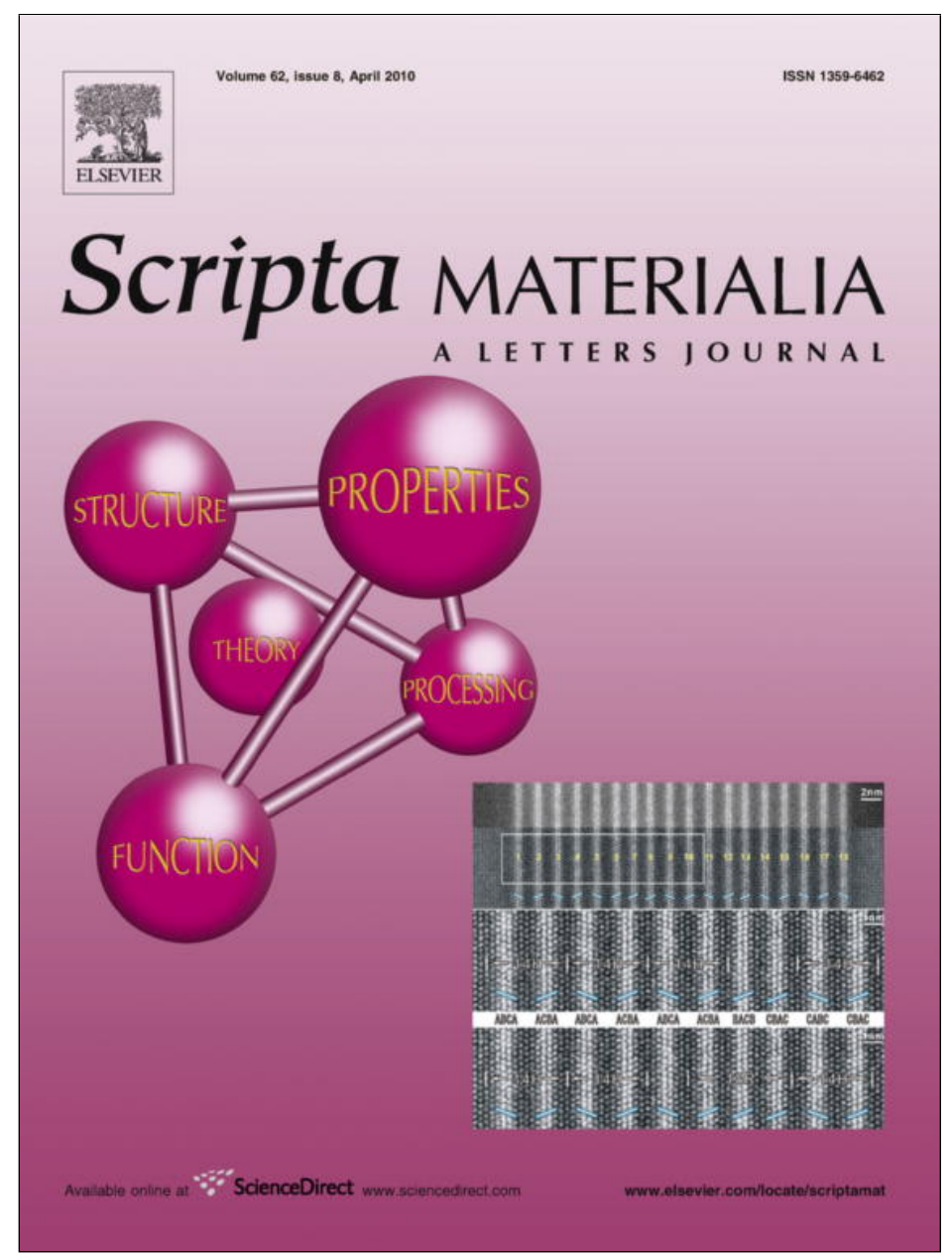

This article appeared in a journal published by Elsevier. The attached copy is furnished to the author for internal non-commercial research and education use, including for instruction at the authors institution and sharing with colleagues.

Other uses, including reproduction and distribution, or selling or licensing copies, or posting to personal, institutional or third party websites are prohibited.

In most cases authors are permitted to post their version of the article (e.g. in Word or Tex form) to their personal website or institutional repository. Authors requiring further information regarding Elsevier's archiving and manuscript policies are encouraged to visit:

http://www.elsevier.com/copyright 


\title{
Nanoscale periodic corrugation to dimple transition due to "beat" in a bulk metallic glass
}

\author{
M.Q. Jiang, ${ }^{\mathrm{a}}$ Z. Ling, ${ }^{\mathrm{a}}$ J.X. Meng, ${ }^{\mathrm{a}}$ J.B. Gao ${ }^{\mathrm{b}}$ and L.H. Dai ${ }^{\mathrm{a}, *}$ \\ ${ }^{a}$ State Key Laboratory of Nonlinear Mechanics, Institute of Mechanics, Chinese Academy of Sciences, \\ Beijing 100190, People's Republic of China \\ ${ }^{\mathrm{b}}$ PMB Intelligence LLC, P.O. Box 2077, West Lafayette, IN 47996, USA
}

Received 3 December 2009; revised 26 December 2009; accepted 26 December 2009

Available online 4 January 2010

\begin{abstract}
We report an intriguing observation that the interaction of brittle nanoscale periodic corrugations (NPCs) can lead to the formation of ductile dimples on the dynamic fracture surface of a tough Vit 1 bulk metallic glass (BMG) under high-velocity plate impact. A "beat" phenomenon due to superposition of simple harmonic vibrations, approximately characterizing NPCs, is proposed to explain this unusual brittle-to-ductile transition. The present results agree well with our previously revealed energy dissipation mechanism in the fracture of BMGs.

(C) 2010 Acta Materialia Inc. Published by Elsevier Ltd. All rights reserved.
\end{abstract}

Keywords: Bulk metallic glasses; Fracture; Energy dissipation; "Beat" phenomenon

The question whether the fracture of bulk metallic glasses (BMGs) is essentially brittle or ductile has puzzled scientists in the recent past [1-6]. Below the glass-transition temperature, BMGs usually suffer a strong tendency for shear localization into ca. $10 \mathrm{~nm}$ thick shear bands [7-13] and undergo macroscopic brittle fracture [14-17]. However, there is clear evidence on the final fracture surfaces that BMGs are capable of legitimate plastic flow at the microscale [18-20]. On the shear-dominated fracture surfaces, microscale cell or river-like vein patterns indicative of typical characteristics of ductile fracture can be widely observed in various BMG systems [14-20]. In particular, the apparently smooth fracture surface of even a brittle La-based BMG can exhibit fine $100 \mathrm{~nm}$ sized dimples, as first reported by Nagendra et al. [21]. Recently, similar dimples and much finer nanoscale periodic corrugations (NPCs) have been found on optically mirror-reflecting fracture surfaces of some ideally or less brittle BMGs [2,4,22-24]. In order to reveal the fracture mechanism, we have performed a systematic study on the fracture patterns of a typical tough $\mathrm{Zr}_{41.2} \mathrm{Ti}_{13.8} \mathrm{Cu}_{10} \mathrm{Ni}_{12.5} \mathrm{Be}_{22.5}$ (Vit 1) BMG under compression, tension and high-velocity plate impact over a wide range of strain rates from $\sim 10^{-4}$ to

\footnotetext{
*Corresponding author. Tel.: +86 10 82543931; fax: +86 10 82543977; e-mail: lhdai@lnm.imech.ac.cn
}

$10^{6} \mathrm{~s}^{-1}[25,26]$. It has been shown that this tough Vit 1 BMG can also exhibit fine dimples at the $100 \mathrm{~nm}$ scale and even NPCs on dynamic mode I fracture surfaces, in addition to conventional vein patterns; these fracture morphologies can interchange with each other with changes in cracking speed and fracture mode.

In accordance with the above findings, we suggest a ductile-to-brittle (DTB) criterion for fracture of BMGs. If the size of the fracture process zone ahead of crack tip, which can be measured by the curvature radius $R$ of the crack tip, is greater than the critical wavelength $\lambda_{c}$ of the fluid meniscus instability $[19,20]$, a fracture with some softening is possible [24-27], as illustrated in Figure 1(a). That relation reads $[20,25]$

$R \geqslant \lambda_{c}=2 \pi\left(\frac{\chi}{\mathrm{d} \sigma / \mathrm{d} x}\right)^{1 / 2}$

where $\chi$ is the surface energy and $\mathrm{d} \sigma / \mathrm{d} x$ is the negative pressure gradient in the front of the crack tip. In this case, atomic cluster in the fracture process zone moves via shear transformation zones (STZs) (see the inset in Fig. 1a), introduced by Argon [28], through which vein patterns or dimples can finally form due to the fluid meniscus instability $[19,20,24-26]$. If the converse is true, i.e. $R<\lambda_{c}$, BMGs must display some "quasi-cleavage" fracture features as a result of continuous tension transformation zones (TTZs) operations [25], as schematized 

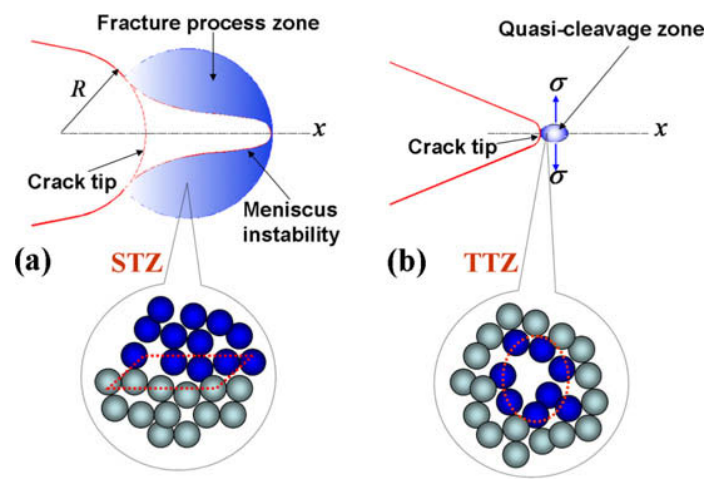

Figure 1. Schematic diagram of the ductile-to-brittle transition mechanism of metallic glasses during fracture. (a) If the curvature radius of the crack tip is greater than the critical wavelength of the meniscus instability, a ductile fracture will occur in the fracture process zone, where atomic clusters move via STZs, as shown in the inset; (b) in the opposite case, local quasi-cleavage fracture through TTZs (see the inset) is possible ahead to the sharp crack tip.

in Figure 1b. The TTZ can be regarded as the counterpart of STZ suffering a significant tension/dilatation but only slight shearing. In other words, TTZs are tension/ dilatation-dominant motions of atomic clusters to alleviate high stress concentrated in the small process zone ahead of the crack tip, whereas STZs are shear-dominant. Due to smaller relaxation timescales, TTZs are less viscoplastic than STZs and more prone to fracture than flow when subjected to stress [5,25]. Through TTZs, stored energy is dissipated mainly by forming new surfaces, resulting in local quasi-cleavage zone ahead of the sharp crack tip. It is important to point out that the crack radius $R$ determining the size of fracture patterns strongly depends on strain rate and ambient temperature. Therefore, even for the same BMG system, it can exhibit the DTB transition phenomenon with varying strain rates $[4,21,25,26]$ or temperatures [5]. The underlying mechanism is the transition between STZs and TTZs ahead of the crack tip. In our previous work we identified that the TTZs with STZs in the background in front of the crack activate periodically, producing NPCs on the fracture surface [25].

In this paper, we show the interesting experimental phenomenon that a ductile dimple can emerge in the region where brittle NPCs prevail on the dynamic fracture plane of a tough Vit 1 BMG undergoing high-velocity plate impact. A "beat" phenomenon due to the interaction of simple harmonic vibrations, approximately describing NPCs, is proposed to explain this transition from brittle NPC to ductile dimple. Such transition confirms our aforesaid DTB criterion for the fracture of metallic glasses.

High-velocity (up to $\sim 500 \mathrm{~m} \mathrm{~s}^{-1}$ ) plate impact experiments on thin Vit $1 \mathrm{BMG}$ disks, $0.3 \mathrm{~mm}$ thick with the diameter of $8.0 \mathrm{~mm}$, were performed using a one-stage light gas gun. The preparations of $\mathrm{BMG}$ specimens and subsequent dynamic experimental process have been detailed previously $[25,26]$. Stress analysis on the finite-diameter disk under a plane shock wave indicates that the crack can propagate in mode I with a strain rate of $\sim 10^{6} \mathrm{~s}^{-1}$ along a radial direction from the edge to the center of the disk. After testing, an FEI Sirion high-res- olution scanning electron microscope (HRSEM) with a spatial resolution of $1.5 \mathrm{~nm}$ and an atomic force microscope (AFM) operated in tapping mode were used to characterize the fracture surfaces of all specimens.

A typical HRSEM image of the apparently "mirrorlike" regions on the dynamic mode I fracture surface of this tough Vit 1 BMGs is presented in Figure 2a. Clearly, fine nanoscale periodic corrugations appear in the "mirror" region. Along the cracking direction, the NPC can evolve into microbranching patterns with increasing crack propagation velocity [26]. However, we found that a dimple at the $100 \mathrm{~nm}$ scale exists in the region predominated by the NPCs, as marked in Figure 2a. The size of the region of dimples is $\sim 1.3 \pm 0.2 \mu \mathrm{m}$ along the propagation direction; NPCs lie on either side of the dimple, as magnified in Figure $2 b$. For convenience, the NPC on the left-hand side of the dimple is denoted by "NPC-1" and that on the right-hand side, "NPC-2". As mentioned above, the dimple formation is due to the fluid meniscus instability within the fracture process zone in front of the crack, when Eq. (1) is satisfied. Therefore, the size of the dimple itself is determined by the dominant wavelength $\lambda_{s}$ of the fluid meniscus instability that is given by [20]

$\lambda_{s}=12 \pi^{2} A(n) \chi / \tau_{y}$

where the constant $A(n)$ is a function of the non-linearity exponent $n$, which ranges from 1 (Newtonian viscous) to 0 (ideal plastic), and $\tau_{y}$ may be taken as the shear yield strength. For the present Vit $1 \mathrm{BMG}[25], A(n) \approx 1.5$, $\chi=0.83 \mathrm{~J} / \mathrm{m}^{-2}$ and $\tau_{y} \approx 1 \mathrm{GPa}$; hence the calculated $\lambda_{s}$ is $\sim 147 \mathrm{~nm}$. This value is in good agreement with
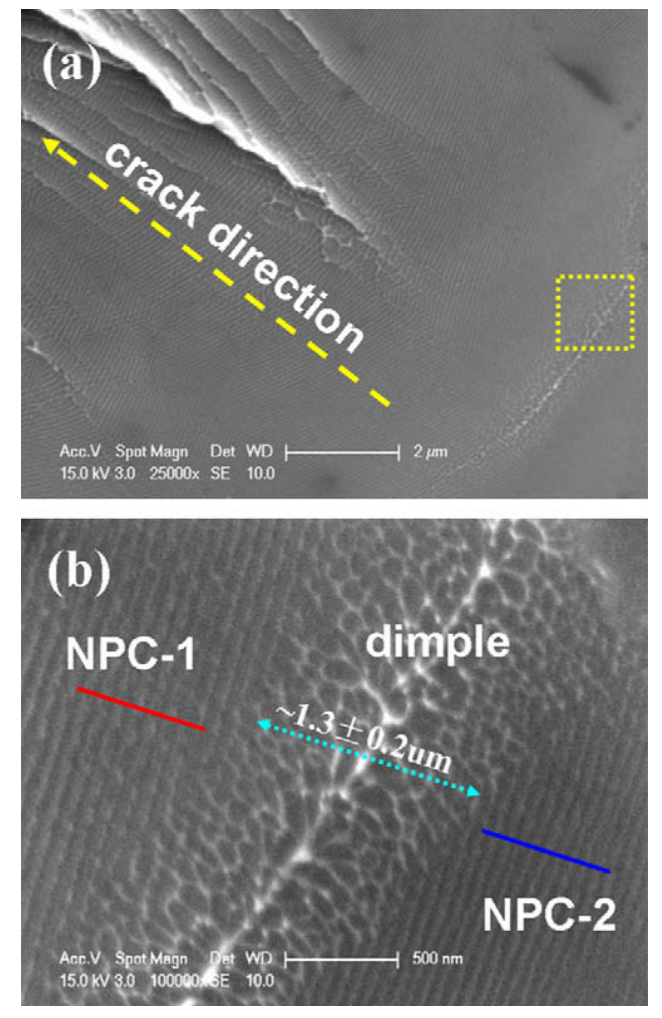

Figure 2. (a) NPC on the dynamic mode I fracture surface of Vit 1 $\mathrm{BMG}$; (b) dimple formation in the NPC-predominated region marked in (a). 

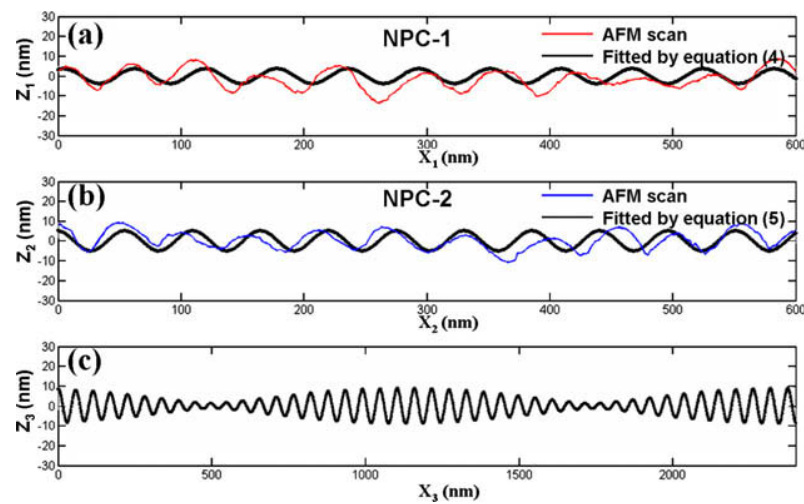

Figure 3. (a) AFM profile of NPC-1 marked in Figure 2(b), fitted by Eq. (4); (b) AFM profile of NPC-2 marked in Figure 2(b), fitted by Eq. (5); (c) superposition of Eqs. (4) and (5) showing a well-known beat phenomenon.

the observed dimple size (see Fig. 2a), implying that the fluid meniscus instability indeed controls the dimple formation. Furthermore, $\lambda_{c}$ can be obtained by the following relationship $[19,20]: \lambda_{c}=\lambda_{s} / \sqrt{3} \approx 85 \mathrm{~nm}$. Considering the projection effects of the SEM, we used an AFM to obtain the precise characteristic size, i.e. spacing, of the NPCs. AFM scans operated in tapping mode were applied to both NPC-1s and NPC-2s. A representative AFM section profile of NPC-1 along its propagation direction, e.g. the red $^{1}$ line in Figure $2 b$, is shown in Figure $3 \mathrm{a}$ as a red curve. Its average spacing, i.e. the peak-to-peak distance, is about $57.80 \mathrm{~nm}$. The section shape of NPC-2 along the blue line in Figure $2 b$ is presented in Figure $3 \mathrm{~b}$ as a blue curve, with an average spacing of $\sim 55.22 \mathrm{~nm}$. The spacing can be regarded as the approximate size of the fracture process zone ahead of the crack tip $[24,25]$. Thus the condition $R<\lambda_{c}$ is satisfied. In this case, the meniscus instability-induced crack propagation is impeded because the fracture process zone is rather small. Alternatively, a local quasicleavage mechanism on the basis of TTZs (see Fig. 1b) has been proposed to explain the NPC formation.

Naturally, some important questions arise. What mechanism is responsible for the transition from brittle NPCs to ductile dimples? Does such DTB transition accord with our proposed criterion? As mentioned previously, alternate activation of TTZs and STZs ahead of the crack tip leads to the arrest (the formation of valley part of NPC) and propagation (the formation of peak part of NPC) of a mode I crack, which gives rise to the NPC formation. During this process, the material point at the crack tip is subjected to a periodic stress and the corresponding deformation or displacement [25]. The NPC extension on either fracture surface can therefore be characterized roughly by the propagation of a simple harmonic vibration expressed by

$Z=A \cos (\omega x+\varphi)$

where $Z$ is the displacement of a material point in the out-of-plane direction, $A$ is the amplitude, $\omega$ is the rota-

\footnotetext{
${ }^{1}$ For interpretation of the references to color in Figures $1-3$, the reader is referred to the web version of this article.
}

tional frequency and $\varphi$ is the initial phase. For the NPC1 , its average spacing or periodicity is about $57.80 \mathrm{~nm}$, corresponding to $\omega_{1}=0.1087 \mathrm{~nm}^{-1}$; its average height or amplitude is $A_{1}=3.78 \mathrm{~nm}$. It can be seen from Figure $3 \mathrm{a}$ that the NPC-1 can be approximately described by the following vibration equation:

$Z_{1}=3.78 \cos \left(0.1087 x_{1}-0.5\right)$

Similarly, according to the AFM scan of NPC-2 (see the blue curve in Fig. 3b), its parameters are $A_{2}=5.13 \mathrm{~nm}$ and $\omega_{2}=0.1138 \mathrm{~nm}^{-1}$. As shown in Figure $3 \mathrm{~b}$, the NPC-2 roughly satisfies the vibration equation written by

$Z_{2}=5.13 \cos \left(0.1138 x_{2}+0.1\right)$

It is noted from Figure $2 b$ that the NPC-1 is almost parallel to the NPC-2. Therefore, the dimple formation is possibly due to the superposition of NPC-1 and NPC2 , which are described by two simple harmonic vibrations, i.e. Eqs. (4) and (5), respectively. Their superposition $Z_{3}=Z_{1}+Z_{2}$ is presented in Figure 3c. Interestingly, a so-called "beat" phenomenon - that is, a periodical change of amplitude - occurs due to the slight difference in the rotational frequencies of the two harmonic vibrations. The amplitude of the spatial coupled vibration $Z_{3}$ has the periodicity $T_{3}$, calculated by

$T_{3}=2 \pi /\left(\omega_{2}-\omega_{1}\right)$

which was calculated to be about $1.2 \mu \mathrm{m}$. This value compares well with the size $(\sim 1.3 \pm 0.2 \mu \mathrm{m})$ of the dimple region, as marked in Figure 2b. If we consider $T_{3}$ as the perturbation length of the meniscus instability, we find that the DTB criterion $T_{3}>\lambda_{c}$ is satisfied; hence the fluid meniscus will be active. In this situation, the material point at the crack tip has the opportunity for being in a lower stress state or having a small vertical displacement for a longer span of time (see Fig. 3c) compared with the dimples formed within the range of $T_{3}$. This transition is essentially due to the increase in the relaxation timescales of TTZs, similar to what Raghavan et al. proposed [5]. One can thus conclude that the dimple formation can be explained by a "beat" phenomenon. It must be pointed out that the occurrence of the "beat" is insensitive to the initial phase of vibrations, depending only on the frequency. If the rotational frequency of NPC-1 is much greater than that of NPC-2, the beat phenomenon will not occur, and vice versa. Strictly speaking, the NPC is quasi-periodic at best (see the AFM profiles in Fig. 3a and b), and can be regarded as the superposition of many simple harmonic vibrations with different inherent frequencies. Their further interaction most probably incurs a disordered vibration with the periodicity approaching infinity. In this case, the NPCs could disappear and evolve completely into dimples and even vein patterns. Although the "beat" phenomenon shown in Figure $3 \mathrm{c}$ is quite simple, it highlights the essential physics that underlies the DTB transition from brittle NPC to ductile dimple, as well as the energy dissipation mechanism for fracture in metallic glasses.

In summary, we observe an unusual fractographic transition from brittle nanoscale periodic corrugations 
to ductile dimples on the seemingly featureless mirror region in a typical tough Vit 1 BMG under high-speed plate impact loading. A "beat" phenomenon due to simple harmonic vibrations with similar frequencies is proposed to explain such transition. The present observation enhances our understanding of the energy dissipation mechanism for BMGs during fracture.

Financial support was from the NSFC (Grants Nos.: 10725211, 10721202, and 10872206), the NSAF (Grant No.: 10976100), the National Basic Research Program of China (Grant No.: 2009CB724401) and the Key Project of Chinese Academy of Sciences (No. KJCX2-YW-M04).

[1] J.J. Lewandowski, W.H. Wang, A.L. Greer, Philos. Mag. Lett. 85 (2005) 77.

[2] X.K. Xi, D.Q. Zhao, M.X. Pan, W.H. Wang, Y. Wu, J.J. Lewandowski, Phys. Rev. Lett. 94 (2005) 125510.

[3] S.J. Poon, A. Zhu, G.J. Shiflet, Appl. Phys. Lett. 92 (2008) 261902.

[4] G. Wang, Y.N. Han, X.H. Xu, F.J. Ke, B.S. Han, W.H. Wang, J. Appl. Phys. 103 (2008) 093520.

[5] R. Raghavan, P. Murali, U. Ramamurty, Acta Mater. 57 (2009) 3332.

[6] X.J. Gu, S.J. Poon, G.J. Shiflet, J.J. Lewandowski, Scr. Mater. 60 (2009) 1027.

[7] T. Masumoto, R. Maddin, Acta Metall. 19 (1971) 725.

[8] P.E. Donovan, W.M. Stobbs, Acta Metall. 29 (1981) 1419.

[9] T.C. Hufnagel, P. El-Deiry, R.P. Vinci, Scr. Mater. 43 (2000) 1071.
[10] J. Li, F. Spaepen, T.C. Hufnagel, Philos. Mag. A 82 (2002) 2623.

[11] Y. Zhang, A.L. Greer, Appl. Phys. Lett. 89 (2006) 071907.

[12] M.Q. Jiang, L.H. Dai, J. Mech. Phys. Solids 57 (2009) 1267.

[13] M.Q. Jiang, W.H. Wang, L.H. Dai, Scr. Mater. 60 (2009) 1004.

[14] Z.F. Zhang, J. Eckert, L. Schultz, Acta Mater. 51 (2003) 1167.

[15] L.F. Liu, L.H. Dai, Y.L. Bai, B.C. Wei, J. Non-Cryst. Solids 351 (2005) 3259.

[16] L.F. Liu, L.H. Dai, Y.L. Bai, B.C. Wei, J. Eckert, J. Mater. Res. 21 (2006) 153.

[17] C.A. Schuh, T.C. Hufnagel, U. Ramamurty, Acta Mater. 55 (2007) 4067.

[18] C.A. Pampillo, J. Mater. Sci. 10 (1975) 1194.

[19] F. Spaepen, Acta Metall. 23 (1975) 615.

[20] A.S. Argon, M. Salama, Mater. Sci. Eng. 23 (1976) 219.

[21] N. Nagendra, U. Ramamurty, T.T. Goh, Y. Li, Acta Mater. 48 (2000) 2603.

[22] Z.F. Zhang, F.F. Wu, W. Gao, J. Tan, Z.G. Wang, M. Stoica, J. Das, J. Eckert, B.L. Shen, A. Inoue, Appl. Phys. Lett. 89 (2006) 251917.

[23] J. Shen, W.Z. Liang, J.F. Sun, Appl. Phys. Lett. 89 (2006) 121908.

[24] G. Wang, D.Q. Zhao, H.Y. Bai, M.X. Pan, A.L. Xia, B.S. Han, X.K. Xi, Y. Wu, W.H. Wang, Phys. Rev. Lett. 98 (2007) 235501.

[25] M.Q. Jiang, Z. Ling, J.X. Meng, L.H. Dai, Philos. Mag. $88(2008) 407$.

[26] J.X. Meng, Z. Ling, M.Q. Jiang, H.S. Zhang, L.H. Dai, Appl. Phys. Lett. 92 (2008) 171909.

[27] S.V. Madge, T. Wada, D.V. Louzguine-Luzgin, A.L. Greer, A. Inoue, Scr. Mater. 61 (2009) 540.

[28] A.S. Argon, Acta Metall. 27 (1979) 47. 